\title{
Review of: "The effect of geriatric intervention in frail older patients receiving chemotherapy for colorectal cancer: a randomised trial (GERICO)"
}

\author{
Fiammetta Monacelli ${ }^{1}$
}

1 University of Genoa

Potential competing interests: The author(s) declared that no potential competing interests exist.

The present randomized Phase III trial is aimed at assessing the the role of CGA based interventions in a sample of older adults with colorectal 2-4 stage cancer in terms of chemotherapy without dose reduction and delay as compared to control patients submitted to standard oncological care. The topic is highly relevant in the field of oncogeriatric and although the sample size is relatively small, the study is wellconducted and adds knowledge.

There are some criticisms that authors should address in order to improve the manuscript comprehension.

- CGA assessment is described as well as the appropriate reference is included. However, it should worth mentioning the CGA cut off in order to stratify patients into fit, pre-frail ( vulnerable) and frail . Frailty stratification in terms of percentage and patients' clinical characteristics should be also included, to better understand the different clinical phenotypes of the heterogeneous set of examined patients. Did fit elders receive higher chemotherapy doses?Exclusion and inclusion criteria need to be better defined. In the discussion, authors claimed that frail and persons with dementia were excluded from the trial but these data were not mentioned in the exclusion criteria. The better definition of the concept of frailty, its cut off along with the cut off for dementia ( which clinical guideline is applied?) should also be included.

- The lower that expected rate of depression needs to be further addressed. It is real surprising that only $6 \%$ of older adults with cancer experienced such a common and disabling clinical condition.

- The primary endpoint stated that CGA based interventions older adults with cancer concluded the planned dose of chemotherapy without any dose reduction and /or delay as compared to controls. However, a bit later in the dose reduction, delays and receive d chemotherapy paragraph, authors stated that no difference occurred in delays that occurred equally in both. This results may be misleading and need to be clearly addressed.

- The authors should comment on the starting reduction dose of chemotherapy of $60 \%$ in both groups . it sounds to the reader as if both CGA assessment and oncological assessment could intercept vulnerable patients.

- Authors should also comment on the similar rate of hospitalization and/or toxicity in both group of patients. These are key relevant oncological endpoints and these endpoints need to be better addressed 
in the discussion along with the prognosis. It seems that apart from immediate clinical benefit ( mobility, burden of illness and quality of life) no substantial difference in the main clinical outcomes is observed.

- conclusions need to underscore potential challenges and future directions in this very important field. 